\title{
Experimental Verification of Induction Phenomenon on Telecommunication Lines by Applying Its Occurrence Mechanisms Using an Artificial ELF Source Generator
}

\author{
Sangmu Lee ${ }^{1} \cdot$ Yoon-Myoung Gimm ${ }^{2} \cdot$ Changsoo Eun ${ }^{3}$
}

\begin{abstract}
In this paper, an electromagnetic induction on a telecommunication line by the distribution line of a power provision system or a feeder line of an electrified railway system has been verified through experiments. The basic cause of induction occurrence by these practical power provision systems is the returning current through the earth. This principle has been confirmed by the experiments documented in this paper which implemented these mechanisms to incur an induction. Experimental methods were used to produce the returning current through the earth. The experiment to find a relationship between inducing strength and the distance between the two phase lines in a power provision line has also been included to confirm that, when the distance is enlarged, the induction effect increases as the crossnullification effect of magnetic fluxes decreases. An experiment for the existence of a shielding effect by another conduction length material has been addedas a protection measure against the induction.
\end{abstract}

Key words : Induction, Coupling, Earth, Electromagnetic Field, Induction Occurrence Mechanism, Shielding Effect.

\section{I . Introduction}

The induction phenomenon in Faraday's law is a wellknown principle used in the various industrial fields (for example, motor circulation, metallic heating, electric transformer) with beneficial applications. Counter measures are put into practice to protect telecommunication services against an induced voltage due to the coupling of electromagnetic fields by a commercial AC power line or the feeder line of a traction system. A question that might be raised is whether the enforced voltage into telecommunication lines by a nearby power line in the fields is really due to the induction phenomenon $[1,2]$. Experiments have been conducted to verify that the interference (electromagnetic force) on telecommunication lines located near power lines is due to the induction coupling. This was done by using an artificially-made source generator and implementing the circuit configurations of induction occurrence mechanisms.

The main configurations of experiments to implementthe induction occurrence mechanisms can be classified into three types: 1) The separation distance between the hot line and the feedback line of the source generator, 2) Adding earthpoints to the feedback line, and 3) Using the ground itself as a feedback line. We conducted our experiment by changing the source level of voltage or current and also by changing the separation distance between the power line and the telecommunication line while going through 1), 2), 3) above.

\section{II . Application of Induction Occurrence Mechanism}

\section{2-1 Principle Consideration}

\section{2-1-1 Induction Generation}

In an $\mathrm{AC}$ power provision, a nearby conductive cable comes to have an electromagnetic force that opposes the change of the magnetic flux crossing. This can be seen in Faraday's law as represented in the equation (1) and Fig. 1.

$$
\boldsymbol{e}=-\frac{\boldsymbol{d} \Phi}{\boldsymbol{d} \boldsymbol{t}}[\boldsymbol{V}]
$$

$e$ : Electromagnetic motive force(induced voltage) [V] $\Phi$ : The quantum of magnetic flux interlink $[\mathrm{Wb}]$

\section{2-1-2 Cross-Nullification of Magnetic Fluxes}

The power line is composed of two hot lines feeding electricity and a feedback line as a return path of con-

Manuscript received August 25, 2010 ; revised December 6, 2010. (ID No. 20100825-028J)

${ }^{1}$ Korea Electronics and Telecommunications Research Institute, Daejeon, Korea.

${ }^{2}$ Dankook University and EMF Safety Inc., Gyeonggi, Korea.

${ }^{3}$ Information and Communication System, Chungnam National University, Daejeon, Korea.

Corresponding Author : Sangmu Lee (e-mail : sangmu@etri.re.kr) 


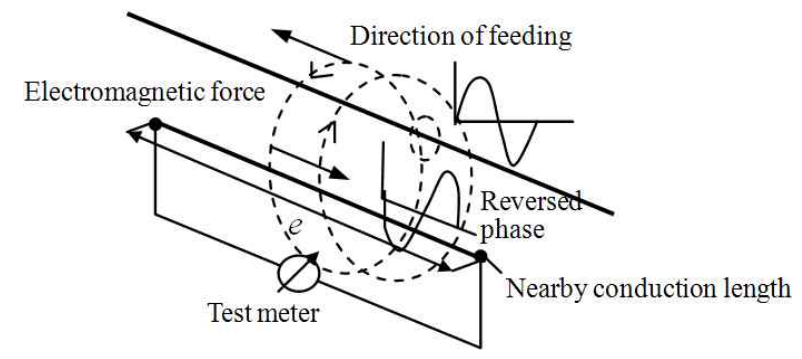

Fig. 1. The electromagnetic motive force generation opposing the change of source current by the induction phenomenon.

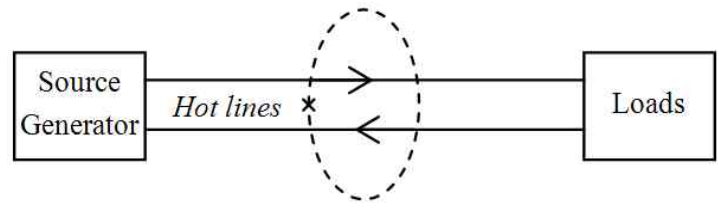

Fig. 2. The opposite direction of AC current flowing by hot lines.

sumed power as shown in Fig. 2. Each hot line has anopposing direction of flow to the other. In a real power provision line, they're adjacent and located close together. This means that the magnetic fluxes of each line are nullified so that they have no inductive effect on other conductors such as nearby telecommunication lines.

\section{2-1-3 The Consideration of Separation between the Hot Lines}

The quantum of interlinks to an induced line by twohot lines changes if distance separates the lines (Fig. 3). The induced line receives an induction when the quantum difference of magnetic flux interlinks to itself and is not nullified due to the distance $x$.

\section{2-2 Practical Situations}

The electromagnetic induction occurs due to the situation of practical fields showed in a Fig. 4.

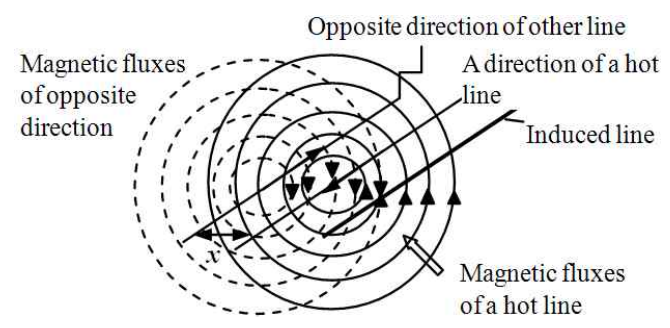

Fig. 3. The influence of the distance, $x$, which retains the inducing effect with the remaining fluxes by the difference of proximity of each line to the induced line.

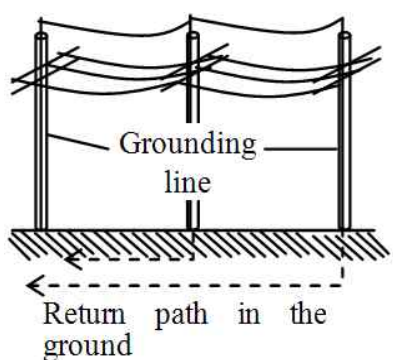

(a) Distribution power line

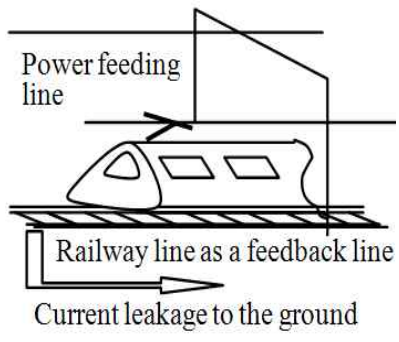

(b) Traction system structure
Fig. 4. The practical situations of returning current through the earth, which is a basic cause of induction.

The (a) case shows the induction source in the distribution line of power provision with the return current in the ground. Case (b) shows the induction source that can occur in the railway system due to the returning current to the earth leaking because of the contact of the rail with the soil. Induction equal to the quantity of the leakage occurs because the cross-nullification effect can not generate enough to cancel out the current leakage.

\section{Performance of Experiment}

\section{3-1 Constellation of Components}

Two circuitry methods were added to our experiments to imitate the practical situations presented in the Fig. 4. One of them has an earth point at each end of the feedback line as marked in Fig. 5. The other one has no feedback line, but the return current will route wholly through the ground as shown in Fig. 6.

In these configurations, the separation distance, $X$, between the hot line and the feedback line is another important variable of this experiment. It is presumed that the induction effect will increase as the distance increases. The separate distance, $D$, between the hot line and the telecommunication line will be varied at each different distance $X$.

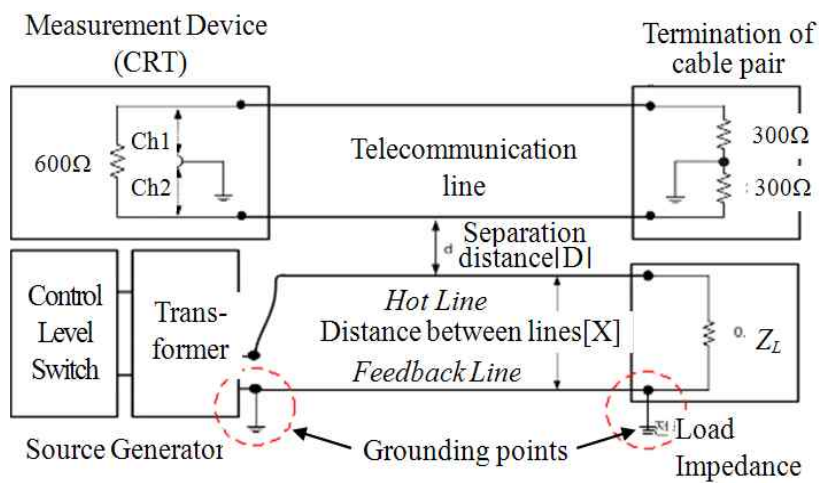

Fig. 5. Adding ground return points for simulation. 


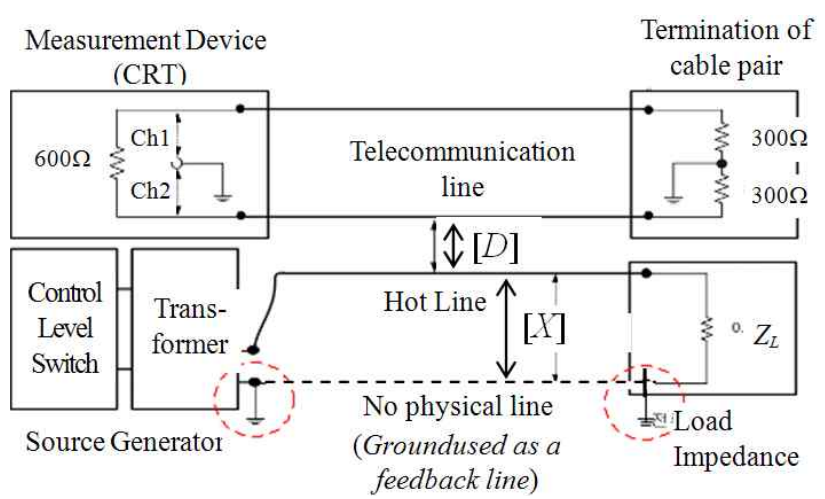

Fig. 6. Using the ground as a feedback route.

\section{3-2 Variable Dispositions}

The change of induced voltage is measured as a process of each variable, taken in steps like Table 1. The source is set up so that $100 \mathrm{~A}$ current will flow.(The practical current flowing in the case of Fig. 6 is $1.6 \mathrm{~A}$, although the source level is set as $100 \mathrm{~A}$ ). The values of 'Ch 1' are used because the 'Ch 2' values are almost the same [3, 4].

Table 1. The experiment variable disposition.

$[\mathrm{m}]$

\begin{tabular}{|c|c|c|c|c|c|c|c|c|c|c|c|c|}
\hline$E$ & \multicolumn{4}{|c|}{ Float } & \multicolumn{4}{c|}{ Leakage } & \multicolumn{4}{c|}{ Ground } \\
\hline$X$ & 1 & 2 & 5 & 10 & 1 & 2 & 5 & 10 & 1 & 2 & 5 & 10 \\
\hline \multirow{4}{*}{$D$} & 0.0 & 0.0 & 0.0 & 0.0 & 0.0 & 0.0 & 0.0 & 0.0 & 0.0 & 0.0 & 0.0 & 0.0 \\
\cline { 2 - 13 }$y$ & 0.3 & 0.3 & 0.3 & 0.3 & 0.3 & 0.3 & 0.3 & 0.3 & 0.3 & 0.3 & 0.3 & 0.3 \\
\cline { 2 - 12 } & 0.5 & 0.5 & 0.5 & 0.5 & 0.5 & 0.5 & 0.5 & 0.5 & 0.5 & 0.5 & 0.5 & 0.5 \\
\cline { 2 - 12 } & 1.0 & 1.0 & 1.0 & 1.0 & 1.0 & 1.0 & 1.0 & 1.0 & 1.0 & 1.0 & 1.0 & 1.0 \\
\hline
\end{tabular}

Float without grounding points in Fig. 5 was also included to see the effect only by the change in X. Leakage corresponds to Fig. 5. Ground corresponds to Fig. 6.

\section{Analysis of Induction Effect}

\section{4-1 Result Measurement Data}

The resulting induced voltage values are showed in Table 2.

\section{4-2 Induction Effect by the Change of ' $X$}

By changing the $X$ in the experiment circuit, we found the data corresponding to the constellation of results seen in Fig. 2 (including the float case). The changes of induced voltage according to the change in $X$ in each $D$ state for the case of float are represented below in Fig. 7.
Table 2. The resulting induced voltages.

[V]

\begin{tabular}{|c|l|l|c|c|c|}
\hline$D[\mathrm{~m}]$ & $X[\mathrm{~m}]$ & 1 & 2 & 5 & 10 \\
\hline \multirow{4}{*}{0.0} & Float & 1.134 & 1.164 & 1.292 & 1.481 \\
\cline { 2 - 6 } & Leakage & 1.128 & 1.307 & 1.536 & 1.872 \\
\cline { 2 - 6 } & Ground & 4.120 & 4.130 & 4.140 & 4.100 \\
\hline \multirow{4}{*}{0.3} & Float & 0.645 & 0.792 & 0.925 & 1.125 \\
\cline { 2 - 6 } & Leakage & 0.727 & 0.864 & 1.089 & 1.290 \\
\cline { 2 - 6 } & Ground & 4.120 & 4.150 & 4.150 & 4.110 \\
\hline \multirow{4}{*}{0.5} & Float & 0.573 & 0.664 & 0.793 & 1.014 \\
\cline { 2 - 6 } & Leakage & 0.630 & 0.714 & 0.937 & 1.175 \\
\cline { 2 - 6 } & Ground & 4.150 & 4.110 & 4.120 & 4.130 \\
\hline \multirow{3}{*}{1.0} & Float & 0.511 & 0.666 & 0.675 & 0.898 \\
\cline { 2 - 6 } & Leakage & 0.588 & 0.672 & 0.876 & 1.056 \\
\cline { 2 - 6 } & Ground & 4.160 & 4.160 & 4.120 & 4.130 \\
\hline
\end{tabular}

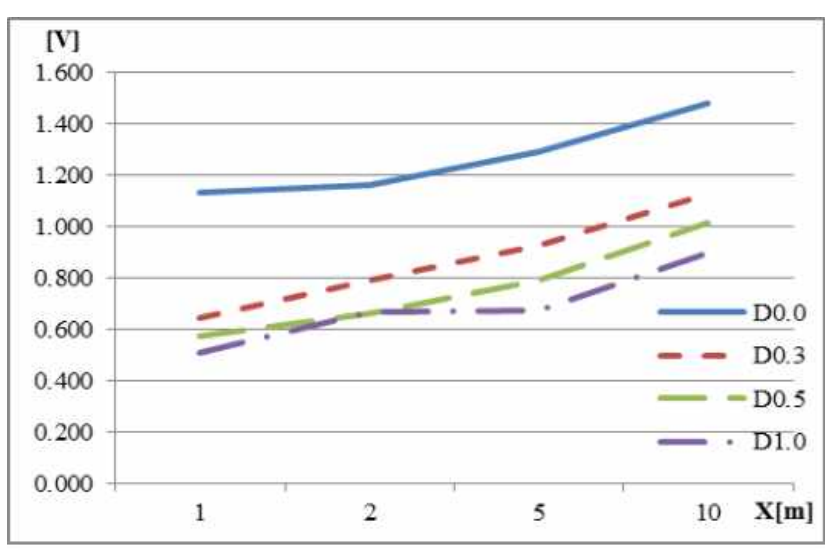

Fig. 7. Changes by $X$ in the case of 'Float'.

In each $D$ case, the voltage is increasing as $X$ is increasing. The level of voltage becomes higher as the $D$ becomes smaller. For leakage, the variation by $X$ is represented in Fig. 8 below. The voltage increases as $X$ increases. The level of voltage is higher when $D$ is smaller.

Comparing Fig. 7 and Fig. 8, the difference in the voltage levels for float and leakage becomes greater as $D$ increases. The induced voltage in the case of leakage becomes much higher than that of float as it did when $X$ was increased. From the sephenomena, it can be said that the induction effect will be more apparent when $D$ is smallest and when $X$ is largest, in other words, when the cross-nullification of the magnetic flux interlinks is smallest.

In the case of ground, the data shows that $X$ does nothave an effect because there is not a physical line for a cross-nullification. It has an induction effect by only a single hot line. 


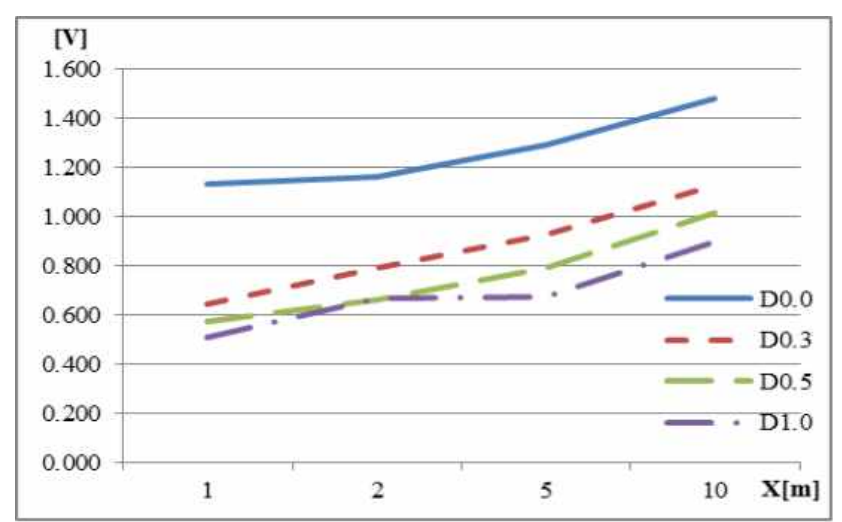

Fig. 8. Changes by $X$ in the case of 'Leakage'.

\section{4-3 Induction Effect with Earth Return Points}

In Table 3 below, $O X$ represents the situation where only $X$ varies. $E X$ represents the case that includes an earth return leakage. $\Delta$ is the difference of $E X$ to $O X$ to show an enhancement by the earth return leakage. ER represents the average values of all $X$ cases on each $D$ case when the earth is used as a return path.

It is shown that $E X$ is about $0.4 \mathrm{~V}$ greater than $O X$ at $D=0$ and $X=10$ as the maximum status, $0.2 \mathrm{~V}$ greater at $D=0$, and $0.12 \mathrm{~V}$ greater for all $D$ 's.

Fig. 9 shows the total scale of differences among the mechanisms with the characteristics of each separation distance $(X$ and $D)$. The lower part of the graph is the voltage area. Around $1.5 \mathrm{~V}$ represents the compared groups of mechanisms that are the cases of only $X$ variation $(O X)$ and $X$ variation with the earth $\operatorname{return}(E X)$. Each group corresponds to each variation of $X$. It also contains the variations according to the changes in $D$.

The induced voltage levels of $O X$ and $E X$ are shown as a pair for comparison on each case of $D$. You can see at a glance that the induced voltage level of $E X$ is slightly higher than that of $O X$ in each pair, the induced voltage decreases as $D$ increases, and they increase as $X$ increasesas shown in the trend of groups. The induced voltages for the complete earth return $(E R)$ are comparatively at a much higher level over 4 voltages. This is around 4 times the maximum values of other mechanisms, which are usually $1 \mathrm{~V}$ or more. The strongest induction effect appeared in this case since the quantum of electromagnetic flux cross-links almost perfectly. The hot line current is maximized.

\section{The Experiment of Shielding Effect as for a Protection Measure against an Induction Interference}

An experiment was performed as shown in Fig. 10, where another conduction length material (see Fig. 11) was placed between the hot line and the telecommunication line (see Fig. 5 and 6). The result was compared to the state without the inserted conduction length material.

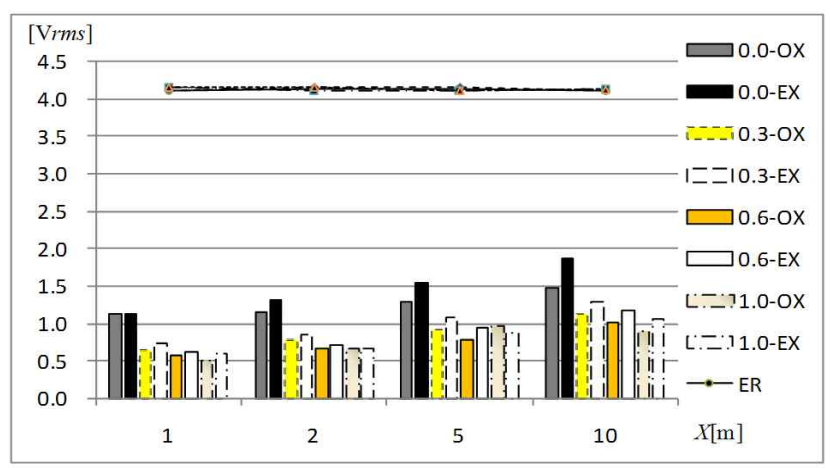

Fig. 9. Total scale view of mechanisms.

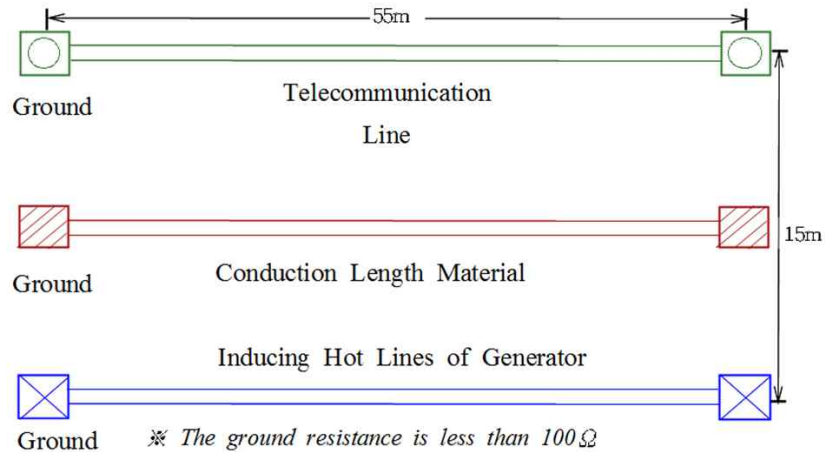

Fig. 10. The arrangement of the conduction material.

Table 3. Comparison between induction mechanisms.

$[\mathrm{Vrms}]$

\begin{tabular}{|c|c|c|c|c|c|c|c|c|c|c|c|c|}
\hline$D$ & \multicolumn{3}{|c|}{$0 \mathrm{~m}$} & \multicolumn{3}{c|}{$0.3 \mathrm{~m}$} & \multicolumn{3}{c|}{$0.6 \mathrm{~m}$} & \multicolumn{3}{c|}{$1 \mathrm{~m}$} \\
\hline$X \mathrm{~L}$ & OX & EX & $\Delta$ & OX & EX & $\Delta$ & OX & EX & $\Delta$ & OX & EX & $\Delta$ \\
\hline $1 \mathrm{~m}$ & 1.13 & 1.13 & 0.00 & 0.65 & 0.73 & 0.08 & 0.57 & 0.63 & 0.06 & 0.51 & 0.59 & 0.08 \\
\hline $2 \mathrm{~m}$ & 1.16 & 1.31 & 0.15 & 0.79 & 0.86 & 0.07 & 0.66 & 0.71 & 0.05 & 0.67 & 0.67 & 0.00 \\
\hline $5 \mathrm{~m}$ & 1.29 & 1.54 & 0.25 & 0.92 & 1.09 & 0.17 & 0.79 & 0.94 & 0.15 & 0.98 & 0.88 & -0.10 \\
\hline $10 \mathrm{~m}$ & 1.48 & 1.87 & 0.39 & 1.13 & 1.29 & 0.16 & 1.01 & 1.18 & 0.17 & 0.90 & 1.06 & 0.16 \\
\hline AVG. & 1.27 & 1.46 & 0.20 & 0.87 & 0.99 & 0.12 & 0.76 & 0.87 & 0.11 & 0.77 & 0.80 & 0.04 \\
\hline ER & \multicolumn{3}{|c|}{4.12} & \multicolumn{3}{|c|}{4.13} & & 4.13 & & & 4.14 \\
\hline
\end{tabular}




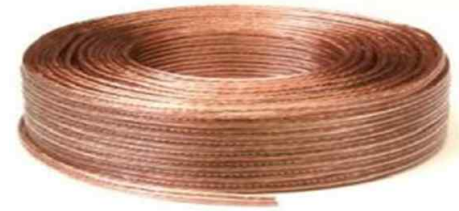

Fig. 11. A conduction length material for a shielding effect.

Table 4. Comparison results from the existing conduction material.

\begin{tabular}{|l|c|c|c|c|c|}
\hline \multirow{2}{*}{$\begin{array}{c}\text { Return } \\
\text { path }\end{array}$} & $X$ & $D$ & \multicolumn{2}{|c|}{$S_{i}$} & \multirow{2}{*}{$k_{i}$} \\
\cline { 2 - 5 } Float & 10 & 0.0 & 1.481 & 1.279 & 0.864 \\
\hline Leakage & 10 & 0.0 & 1.872 & 1.550 & 0.828 \\
\hline Ground & 1 & 0.0 & 4.120 & 3.250 & 0.789 \\
\hline
\end{tabular}

The comparison results are shown in Table 4 .

The conditions for the data shown are $D=0$ and $X=10$ (but this is not meaningful when the feedback current is through the ground), and the source is $100 \mathrm{~A}$ for $\mathrm{Ch} 1$. (which is most effective). The induced voltage of each return path when the conductive length material was used is lower than when the material wasn't used. From this result, we know that some conductive material can protect against the induced voltage on telecommunication lines by a power line.

The ratio of the with material voltage to the without material voltage becomes a shielding coefficient, $k_{i}$. The $k_{i}$ is 0.864 for float, 0.828 for leakage, and 0.789 for ground. The averaged coefficient, $k_{i a}$, is 0.827 . This coefficient means that the induced voltage is lessened as $\left(1-k_{i}\right) \times 100 \%$.

\section{Conclusion}

This paper provides the extended results of induction phenomenon that were dealt with as a basic confirmation of existence at [4]. The experimentsin this paper have been conduced by applying the following practical induction occurrence mechanisms: 1) Enlarging the distance between two hot lines in a power line, 2) Adding a grounding point at the load position to make a return current leakage to the earth, and 3) Using the ground to flow the return current through the earth without using a physical conductor.

Our results confirmed that the induced voltage in- creases as the distance between two hot lines $(X)$ increases. The induction effect is greatest when $X$ is largest, at the smallest separation between the hot line and the telecommunication line $(D)$. The induced voltage can be strengthened even more when a returning current path through the earth exists and is made greater by a mechanism from 2) or 3).

We have also verified that an induction can occur in the real fields of power provisions (the distribution line of a commercial power system or the feeder line of a traction system) by applying the generating mechanisms from the experiments. The induction effect can be more powerful when the system environment is configured so as to make the cross-nullification of each hot line's magnetic fluxes least. Another conduction length material can be used to reduce the amount of induced voltage in order to protect a telecommunication line.

This research was supported by the ICT Standardization program of MKE(The Ministry of Knowledge Economy).

\section{References}

[1] S. G. Yeo, C. W. Park, and J. T. Kim, "A study on causes generating induced noise voltage on telecommunication cables near to high-speed rails," JKSR.11, 248-256, Jun. 2008.

[2] S. G. Yeo, C. W. Park, "A study on the generation of the earth potential and communication line noise," JKIIEE21, pp. 181-188, Dec. 2007.

[3] S. M, Lee et al., "Characteristics of the induced voltages between the communication lines and ground by the adjacent artificial high voltage or current ELF source," 2009 Korea-Japan Joint Conference on $A P / E M C / E M T$, pp. 199-202, May 2009.

[4] S. M. Lee et al., "Characteristics of the voltages between the communication lines and ground induced by the adjacent artificial high voltage or current ELF source," JKIEES, pp. 175-181, Dec. 2009.

[5] Low Frequency Interference due to Unbalance about Earth of Telecommunication Equipment, K. 10, ITUT, Oct. 1996.

[6] Induction, Tokai Sector of Telecommunication Association of Japan, Dec. 1978. 


\section{Sangmu Lee}

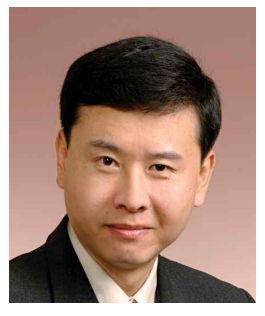

received the B.E. degree in electronics engineering from Dankook University, Korea, in 1989, and the M.E. degree in computer engineering from Information and Communications University, now Korea Advanced Institute of Science and Technology - IT Convergence Campus in 2000. He completed the course work of information and telecommunication system engineering for $\mathrm{Ph}$. Degree at Chungnam National University. He has been working in the Electronics and Telecommunications Research Institute of Korea since 1991. His main researching role is to make an establishment of technical regulation related to electromagnetic induction and protective measure.

\section{Yoon-Myoung Gimm}

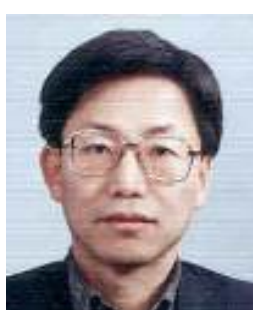

received the B.E. degree in electronics engineering from the Seoul National University, Seoul, Korea, in 1975, and the M.E. and Ph.D. degrees in electrical and electronics engineering from Korea Advanced Institute of Science and Technology (KAIST) in 1977 and 1990, respectively. Since 1980, he has been with the department of electronics engineering, Dankook University, Seoul, Korea, where he is now a professor in the school of electronics and computer engineering. He is also a CEO of EMF Safety Inc., since 2000. His research interests are the electromagnetic field measurements related with the biological effect.

\section{Changsoo Eun}

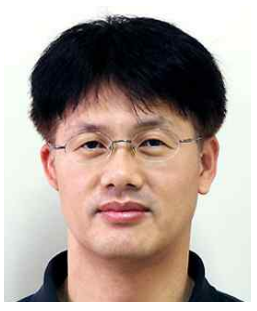

received his B.S. and M.S. degrees from Seoul National University in 1985 and 1987, respectively. He got his Ph.D. degree from the University of Texas at Austin in 1995. From 1987 to 1997, we worked for Daewoo Electronics Co., Seoul as a senior researcher. From 1997, he has been working with Chungnam National University as a faculty member. His research interest includes signal processing for telecommunications, RF circuits and applications. 